\title{
Investigaciones sobre la influencia del factor de forma y del rozamiento en procesos de compresión de piezas cilíndricas de aleación de aluminio AA 6082-T6 mediante técnicas numéricas y experimentales ${ }^{(*)}$
}

\author{
M.M. Marín*, A.M. Camacho*, C. Bernal* y M.A. Sebastián*
}

\begin{abstract}
Resumen
La caracterización de los materiales bajo similares condiciones de carga a las que se tienen durante su fabricación permite abordar el estudio de Procesos de Conformado por Deformación Plástica a Compresión (PCDPC) desde un punto de vista más realista. La forja abierta de la aleación de aluminio AA 6082-T6 ha sido sometida a análisis, combinando técnicas experimentales y el Método de los Elementos Finitos (MEF). A través del modelo numérico se ha analizado la influencia de la geometría de la pieza en los efectos que el rozamiento provoca en variables típicas de estos procesos como las presiones aplicadas, presiones de contacto y tensiones y deformaciones en las piezas. Se ha demostrado que se debe elegir una geometría de pieza inicial con un factor de forma lo más elevado posible para minimizar el efecto del rozamiento. Estos resultados son de interés para disminuir la energía requerida en el proceso así como el desgaste de herramientas y podrían extrapolarse a procesos como la estampación.
\end{abstract}

Investigations on the influence of the shape factor and friction in compression processes of cylindrical billets of AA 6082-T6 aluminum alloy by numerical and experimental techniques

\begin{abstract}
The material characterization under similar load conditions to those applied during manufacturing is important in order to analyse Bulk Forming Compression Processes from a more realistic point of view. Open die forging of AA 6082-T6 aluminum alloy has been analysed by using experimental techniques and Finite Elements Method (FEM). The influence of the workpiece geometry on the effects that friction produces in typical process variables such as applied forces, contact pressures and stresses and strains in workpieces has been analysed by FEM simulation. It has been shown that higher shape factors of the initial workpiece geometry are recommended in such a way that friction effect is minimised. These results are interesting in order to decrease the required energy of the process and the tool wear, and could be used in further analysis of stamping processes.
\end{abstract}

Keywords

Shape factor; Friction; Forging; FEM; Compression.

\section{INTRODUCCIÓN}

A lo largo de los últimos años las ventajas de los Procesos de Conformado por Deformación Plástica (PCDP) están resultando cada vez más evidentes, principalmente debido al alto grado de aprovechamiento del material que comportan. Además, en los procesos en frío se suelen conseguir sensibles mejoras en el comportamiento mecánico de los materiales con precisiones geométricas más que suficientes para muchas aplicaciones industriales o para servir de preformas de geometría bastante ajustada para otros procesos de fabricación, tales como el mecanizado. Existe una amplia variedad de PCDP, con tipologías y características tecnológicas muy diferenciadas, tal como se aprecia en la norma DIN $8582^{[1]}$ que tiene por objeto la clasificación de estos procesos de conformado plástico, Grupo Principal 2 Conformar

(•) Trabajo recibido el día 26 de julio de 2012 y aceptado en su forma final el día 28 de febrero de 2013 .

* Departamento de Ingeniería de Construcción y Fabricación, E.T.S. de Ingenieros Industriales, Universidad Nacional de Educación a Distancia. C/ Juan del Rosal, 12 (Ciudad Universitaria) 28040 Madrid, E-Mail: mmarin@ind.uned.es. 
(Umformen en alemán y Forming en inglés). En este estudio únicamente van a ser considerados los procesos en que predomina el fenómeno de compresión (Grupo 2.1 de DIN), que a lo largo del presente trabajo van a ser designados como Procesos de Conformado por Deformación Plástica a Compresión (PCPDC), y en particular el proceso de forja libre 2.1.2.4 (Stauchen y Forging) ${ }^{[2]}$.

En este tipo de procesos se deben considerar la carga de trabajo, características mecánicas del material y parámetros tecnológicos para obtener el resultado deseado. La carga de trabajo determinará la potencia que se requiere y el tamaño del equipo necesario para realizar el proceso. A su vez, ésta misma está relacionada con la fluencia del material ya que si se trabaja a temperatura ambiente, es decir, conformado en frío, como es el caso, la carga máxima va a depender del endurecimiento por deformación que sufre el material ${ }^{[3]}$. Por otro lado, es muy importante conocer cómo se deforma plásticamente el material, el cual fluye por la combinación de tensiones debidas a la aplicación de la carga de compresión ${ }^{[4]}$. Por último, la obtención de la pieza final está condicionada por parámetros tecnológicos como rozamiento, temperatura, geometría inicial de la pieza y geometría de la herramienta, entre otros.

Los procesos de compresión uniaxial de piezas cilíndricas permiten por un lado caracterizar la fluencia del material que se produce en los procesos de conformado de metales y por otro estudiar parámetros tecnológicos como el rozamiento que interviene en el proceso ${ }^{[5-7]}$. Para realizar el conformado a compresión de las piezas se puede emplear una máquina universal de ensayos. En este tipo de proceso, conformado por deformación plástica a compresión mediante matrices planas y paralelas, se requiere realizar algunas consideraciones particulares en los resultados obtenidos debido a la condición de rozamiento que se produce entre las superficies en contacto.

En el presente trabajo se describe una metodología a seguir en el proceso de compresión para minimizar el rozamiento e interpretar de manera adecuada los resultados obtenidos ${ }^{[8-10]}$. El estudio se realiza tanto con ensayos experimentales como con métodos numéricos de análisis. Como herramienta de análisis numérico se emplea el Método de los Elementos Finitos (MEF). Frente a métodos convencionales que suelen considerar un comportamiento del material rígido plástico perfecto, como el Teorema del Límite Superior, muy utilizado en el análisis de una gran variedad de procesos de conformado como el estirado $^{[11]}$, la precisión de las simulaciones numéricas a través del MEF depende en gran medida de la exactitud en la descripción de la caracterización del material $^{[12]}$. Como se ha demostrado en varios estudios, como el desarrollado por Sofuoglu y Gedikli ${ }^{[8]}$, la caracterización del material a través del proceso de compresión con matrices planas paralelas es el más recomendado para su implementación en los modelos que se emplearán en las simulaciones de PCDPC, tales como forja ${ }^{[13]}$, indentación ${ }^{[14]}$ o la Forja Localizada-Incremental (LIF) ${ }^{[15]}$.

A lo largo del estudio se analiza uno de los parámetros tecnológicos más influyentes como es el rozamiento existente entre las superficies en contacto, la geometría de la pieza y la curva $\sigma-\varepsilon$ del material empleado. El estudio de estos elementos se ha realizado a través del MEF, validando los modelos empleados con los ensayos experimentales realizados. Finalmente, la pieza obtenida en el ensayo se ha escaneado mediante un sistema de digitalización 3D, obteniendo de esta manera una mayor precisión de las no uniformidades que se producen junto con sus dimensiones finales, lo que puede utilizarse para ampliar las posibilidades de análisis mediante la aplicación de técnicas de ingeniería inversa.

\section{METODOLOGÍA EXPERIMENTAL}

Para analizar la fluencia del material se han realizado una serie de ensayos experimentales ${ }^{[4]}$ mediante una máquina universal de ensayos Hoytom, modelo HM-D (Fig. 1(a)). Los ensayos han consistido en someter a varias piezas cilíndricas a una determinada carga de compresión uniaxial mediante la acción de dos matrices planas y paralelas (platos de compresión) simétricamente opuestas (Fig. 1(b)).

Durante los ensayos realizados se han registrado las fuerzas de compresión aplicadas y las variaciones en altura de la pieza (alargamiento). Una vez obtenidos los resultados se ha procedido a procesar analíticamente dichos valores y así obtener resultados de tensiones-deformaciones verdaderas.

A medida que tiene lugar el proceso llega un determinado momento en que la deformación de la pieza deja de ser uniforme, dificultando el cálculo de las tensiones producidas. Para solventar este problema, algunos de los ensayos se han realizado con aplicación de la carga de compresión de manera incremental, reduciendo así el rozamiento y siendo capaces de calcular las tensiones aplicando el Método del Factor de Corrección del Abarrilamiento (Bulge Correction Factor Method-BCFM) ${ }^{[16]}$. Para comprobar la influencia del rozamiento en el proceso se han llevado a cabo varios ensayos con aplicación de la carga de manera continua bajo las mismas condiciones de trabajo y así compararlos. En ambos procedimientos se define como límite del ensayo una fuerza axial de compresión de 90 kN. Para todos los ensayos reali- 

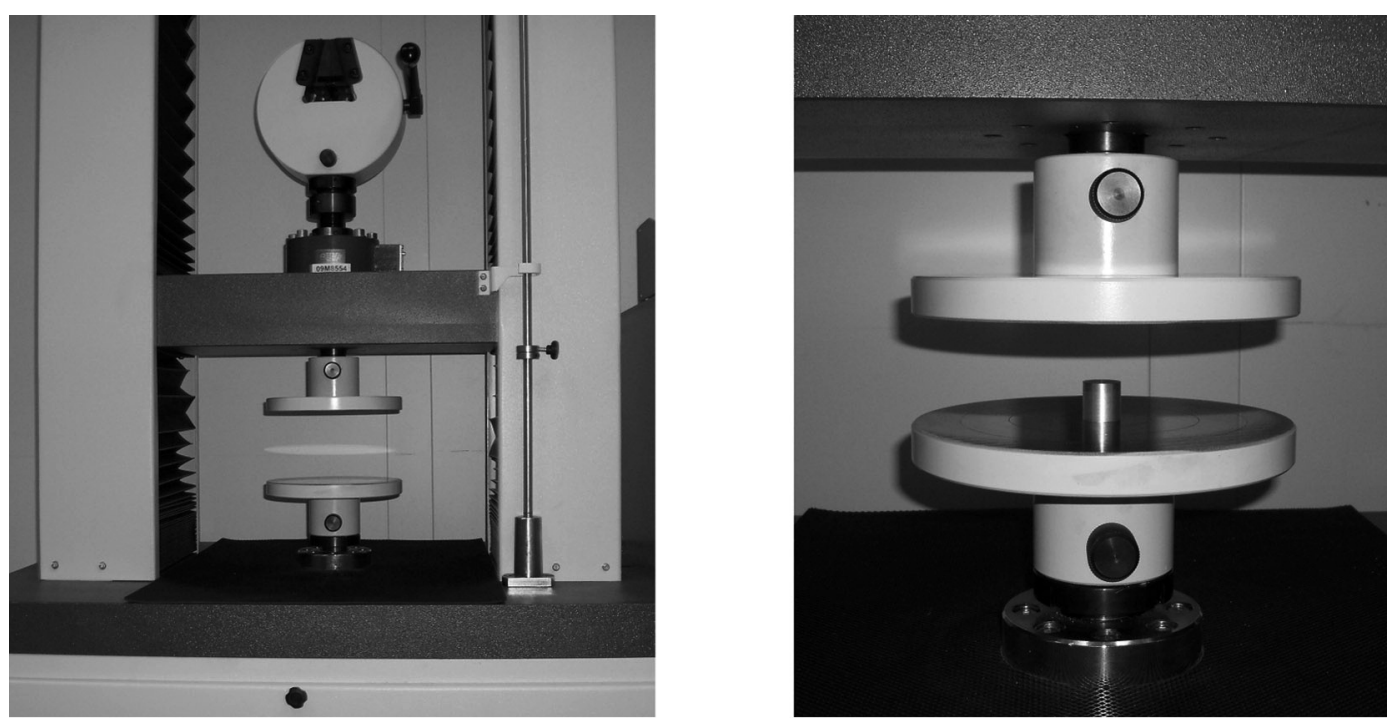

Figura 1. Equipo experimental empleado: a) vista de conjunto; b) detalle de platos de compresión.

\section{Figure 1. Experimental equipment: a) machine overview; b) detail of the compression} plates.

zados se ha utilizado un lubricante a base de grafito para minimizar los efectos del rozamiento existente entre la pieza y los platos de compresión.

\subsection{Metodología incremental y continua}

Se han llevado a cabo varios procesos de compresión mediante dos metodologías diferentes de aplicación de la carga de compresión. En un primer grupo, la aplicación de la carga se ha realizado de manera continua, mientras que en un segundo grupo, la carga se ha aplicado de manera incremental. Todos los procesos, tanto de aplicación de la carga de compresión de manera continua como de manera incremental, se han realizado para diferentes geometrías de pieza y las superficies en contacto han sido lubricadas a base de grafito.

En los procesos de compresión con aplicación de la carga de manera incremental se ha aplicado una carga de compresión hasta que se produce una pequeña deformación plástica. Una vez aplicada y retirada la carga se ha procedido a extraer la pieza para medir sus dimensiones y aplicar el BCFM. Una vez medida y registrados los datos, tanto de fuerza aplicada como de alargamiento, se procede a introducir de nuevo la pieza en la máquina de ensayo para aplicar el siguiente incremento de carga de compresión. Así se realiza sistemáticamente hasta alcanzar una fuerza de compresión de $90 \mathrm{kN}$. En cada incre- mento de carga nuevo se procede a lubricar las superficies en contacto.

En los procesos con aplicación de la carga de compresión de manera continua se introduce la probeta en la máquina de ensayo previamente lubricadas las superficies que están en contacto. Posteriormente se aplica una carga de compresión de 90 kN. Una vez finalizado el proceso se procede a medir la pieza deformada y a registrar los datos de fuerza y alargamiento.

Finalmente, los resultados de fuerzas y alargamiento en cada uno de los casos son tratados para obtener valores de tensión frente a la deformación producida en la pieza.

\subsection{Parámetros tecnológicos más relevantes}

Debido a la gran similitud del estudio con el ensayo de compresión uniaxial para la obtención de la curva de fluencia de un materia ${ }^{[17}$ y 18$]$, es de suponer que los parámetros que influyen en este último también influirán en el estudio realizado. En estudios previos elaborados ${ }^{[3]}$ se ha demostrado que la curva de fluencia de un material depende entre otros, del rozamiento existente entre las superficies en contacto, de la velocidad de deformación y de la temperatura del ensayo. Este último parámetro es aplicable cuando el ensayo se realiza a temperaturas elevadas, donde el material tiene un comportamiento diferente en los rangos de cambio de fase. En el presente estudio 
todos los procesos se han llevado a cabo a temperatura ambiente puesto que los procesos de compresión a analizar son equiparables a procesos de forja abierta en frío.

Como se ha mencionado anteriormente, la curva de fluencia de un material también depende de la velocidad de deformación, aunque se ha demostrado que a temperatura ambiente, prácticamente en todos los metales este parámetro casi no influye ${ }^{[19]}$, por ello en este caso se ha mantenido constante la velocidad de accionamiento del plato de la máquina de compresión siendo ésta de valor $2,5 \mathrm{~mm} / \mathrm{min}$.

Por tanto, bajo las condiciones dadas en los procesos llevados a cabo, el parámetro más influyente es el rozamiento. En el estudio se ha considerado un rozamiento de deslizamiento o de Coulomb variando su valor desde 0,1 hasta 0,5 en incrementos de 0,1.

\subsection{Material}

En el estudio se ha empleado la aleación de aluminio AA 6082-T6 [UNS A96082] cuyos principales aleantes son el magnesio y el silicio. La elección de este tipo de aleación ha sido motivada por su versatilidad y gran utilización en diferentes procesos de fabricación. Es una aleación de alta resistencia (de las más altas de las aleaciones de la serie 6000), por lo que es empleada extensamente en mecanizado. Presenta buenas características para la forja. Es de uso general en la construcción de maquinaria industrial, armamento y blindaje. Se utiliza en el sector del transporte, puentes e ingeniería en general debido a su alta resistencia. Otro de sus principales usos es la fabricación de moldes y matrices para la industria en general.

\subsection{Geometría}

Las piezas sometidas a experimentación tienen diferente geometría; el diámetro se ha mantenido constante a lo largo del estudio mientras que su altura se ha ido cambiando. Las piezas analizadas tienen un diámetro de $10 \mathrm{~mm}$ y diferentes alturas $(5,10 \mathrm{y}$ $20 \mathrm{~mm}$ ) obteniéndose así un factor de forma. El factor de forma se ha definido como la relación entre la altura inicial y el diámetro inicial de la pieza a ensayar. Se han estudiado los siguientes factores de forma $(h / d): 0,5 ; 1$ y 2 , mostrados en la figura 2 .

\subsection{Datos obtenidos por experimentación}

En la figura 3 se muestra la geometría inicial de las piezas junto a la geometría final obtenida para procesos realizados con diferente factor de forma y diferente metodología de aplicación de la carga.

Como se observa en la figura 3 , en las piezas obtenidas con aplicación de la carga de manera incremental se aprecia un menor efecto de abarrilamiento debido a que se minimiza el efecto del rozamiento, al permitir una lubricación más efectiva entre las superficies en contacto, lo que revierte además en la

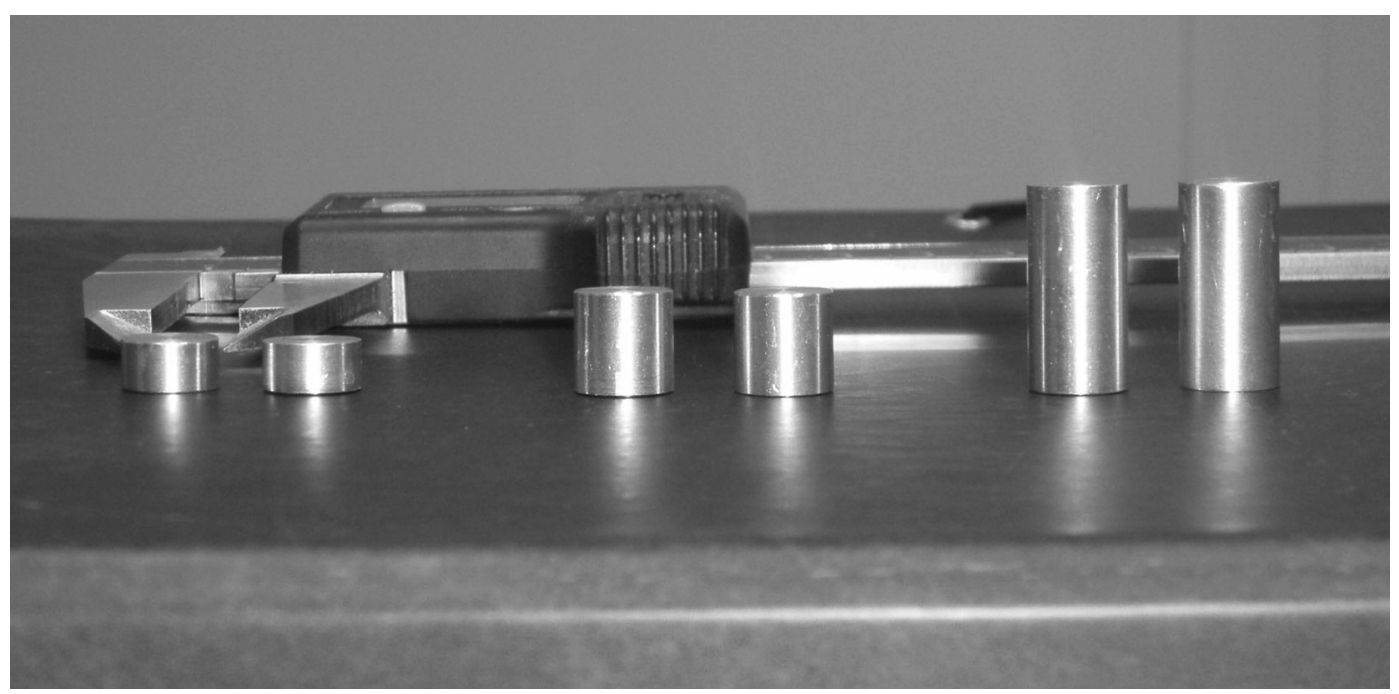

Figura 2. Geometría inicial de las piezas estudiadas con factores de forma (h/d): 0,$5 ; 1$ y 2 .

Figure 2. Initial geometry of the workpieces with shape factors (h/d): 0.5; 1 y 2 . 
posibilidad de alcanzar mayores grados de deformación final en las piezas.

Por el contrario, en el caso del ensayo con aplicación de la carga de manera continua se muestra un mayor abarrilamiento. Con esta geometría final de pieza se ha demostrado en varios estudios que se puede calcular el rozamiento de semiadherencia existente entre las superficies de contacto[6].

Para una correcta determinación del factor de corrección del abarrilamiento definido en ${ }^{[16]}$ es necesario calcular el radio de abarrilamiento. No obstante, dado que la pieza real deformada contiene ciertas irregularidades superficiales y puede resultar algo complicado determinar con exactitud algunas de las dimensiones que intervienen en su cálculo, resulta más adecuado recurrir a técnicas de escaneado tridimensional. Una primera solución para la determinación del radio de abarrilamiento es, una vez escaneada la pieza, aproximarlo mediante el radio de la esfera

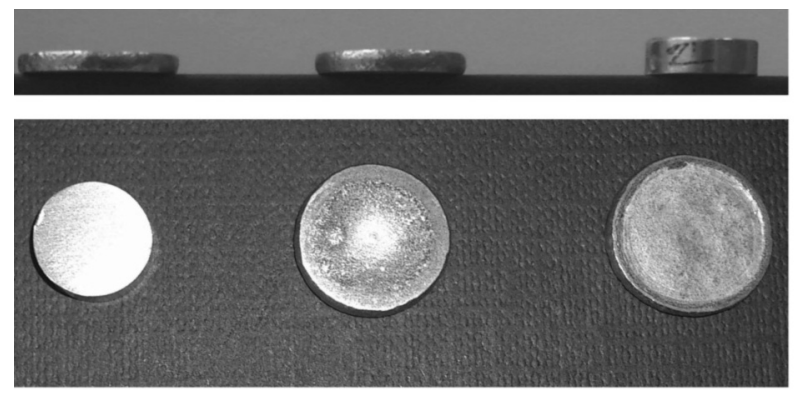

$$
h / d=0,5
$$
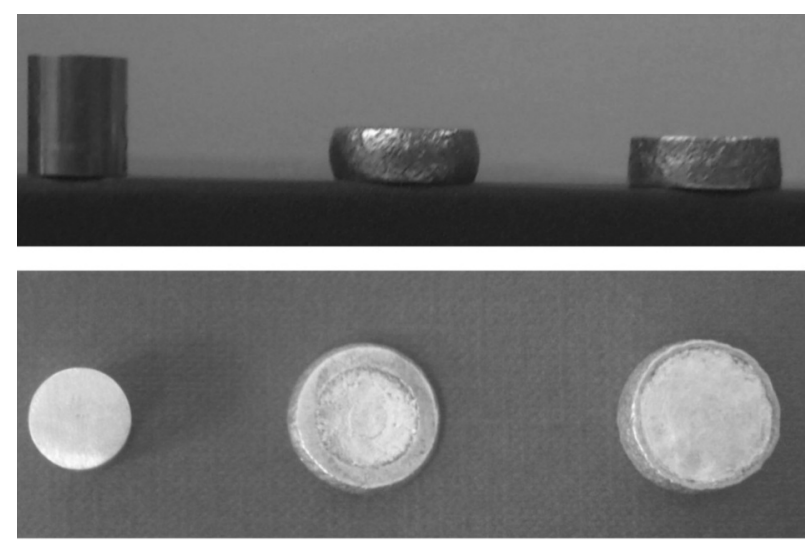

$h / d=1$

Figura 3. Geometría inicial y final de las piezas de ensayos realizados con diferente factor de forma y metodología de aplicación de la carga (centro: continua; derecha: incremental).

Figure 3. Initial and final geometry of the workpiece for different values of shape factor and methodology for applying the load (central: continuous; right: incremental). que mejor se aproxima a la nube de puntos que configuran la geometría tridimensional deformada (Fig. 4).

En la figura 5 se muestran las curvas tensióndeformación obtenidas a partir de los valores de fuerzaalargamiento extraídos de los experimentos realizados en las piezas de la aleación de aluminio AA 6082-T6. Como se ha mencionado anteriormente, los ensayos se han realizado aplicando la carga de manera incremental y continua. En ambos casos, las tensiones y deformaciones han sido calculadas mediante las ecuaciones de tensión y deformación verdadera dentro del campo plástico. En el caso de aplicación de carga incremental, el diámetro y la altura de la pieza son medidos en cada etapa de compresión. A partir del momento en que la deformación deja de ser uniforme se aplica el Método del Factor de Corrección de Abarrilamiento (BCFM). En el caso de aplicación de carga de manera continua, los datos registrados de fuerza y alargamiento en la máquina de ensayo a lo largo de toda la compresión son tratados analíticamente aplicando el principio de conservación de volumen, obteniéndose las deformaciones y tensiones verdaderas. De los diferentes factores de forma de pieza estudiados en la figura 2 se representan los correspondientes a los valores $h / d=1$ y $h / d=2$.

Como se puede observar en las gráficas representadas en la figura 5, las curvas tensión-deformación obtenidas en el proceso llevado a cabo con aplicación de la carga de manera continua presentan diferencias entre ellas al considerar distintos factores de forma. Se alcanzan mayores tensiones para un factor de forma menor, mientras que las curvas obtenidas mediante aplicación de la carga de manera incremental son similares, obteniéndose valores de tensión dentro de un mismo rango. Por otro lado, al observar las gráficas obtenidas con un factor de forma $h / d=1$ se muestra cómo los valores de tensión obtenidos en el proceso incremental son menores que en el proceso continuo alcanzándose deformaciones mayores. Es decir, se minimiza el rozamiento existente entre las superficies en contacto. En el caso del factor de forma $h / d=2$ se obtienen unas curvas muy similares.

Por tanto, la geometría inicial de la probeta influye en la obtención de los valores de tensión y de deformación del proceso, habiendo una relación clara con el rozamiento existente entre las superficies en contacto.

\section{DESARROLLO Y CONTRASTE DEL MODELO NUMÉRICO}

Observada cierta relación entre el factor de forma y los efectos del rozamiento sobre las curvas tensión-deformación del material, se va a tratar de ana- 

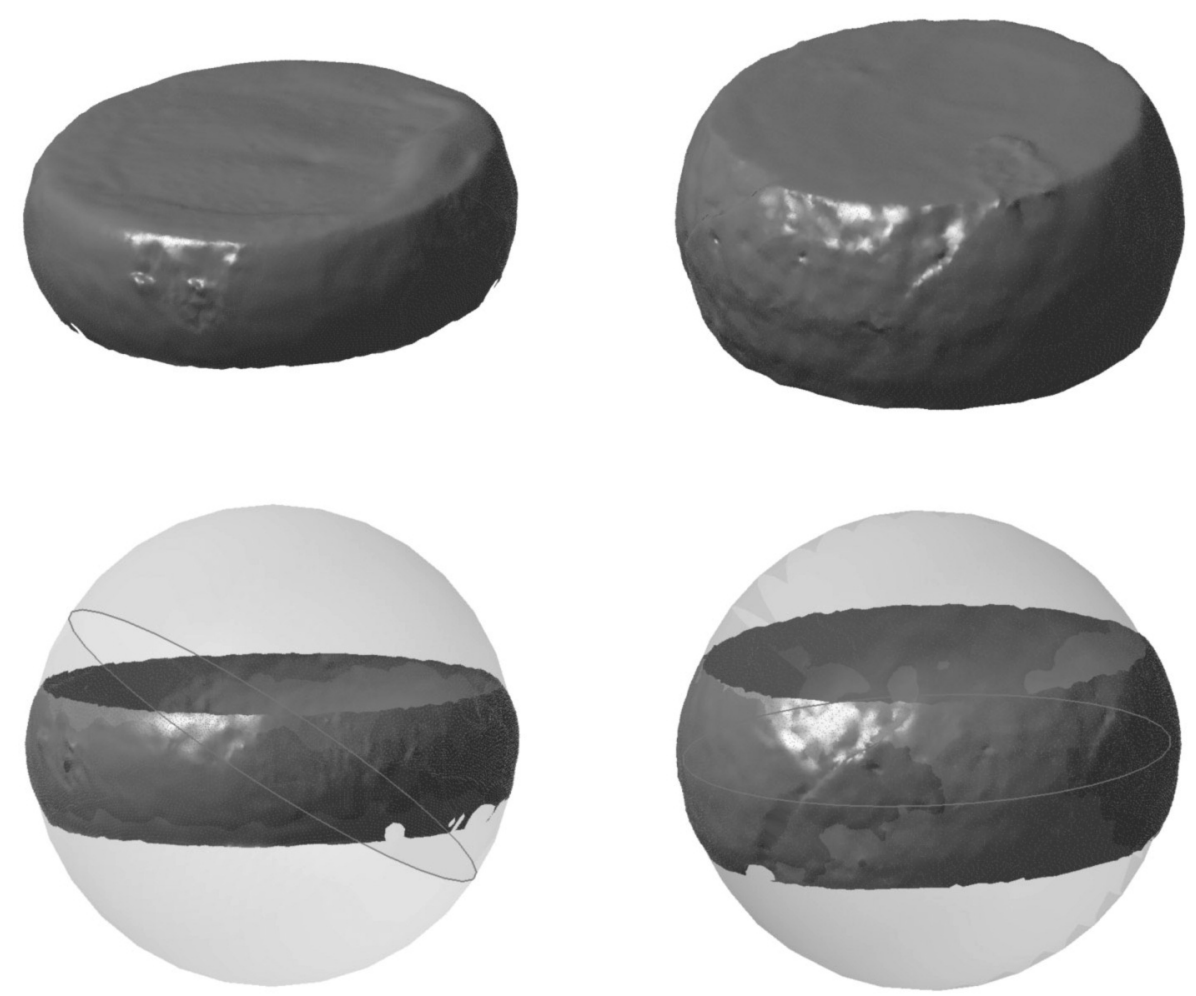

Figura 4. Escaneado tridimensional de la geometría final de la pieza para diferentes factores de forma y determinación del radio de abarrilamiento.

Figure 4. Three-dimensional scanning of the final geometry of the workpiece for different shape factors and calculation method of the bulge radius.

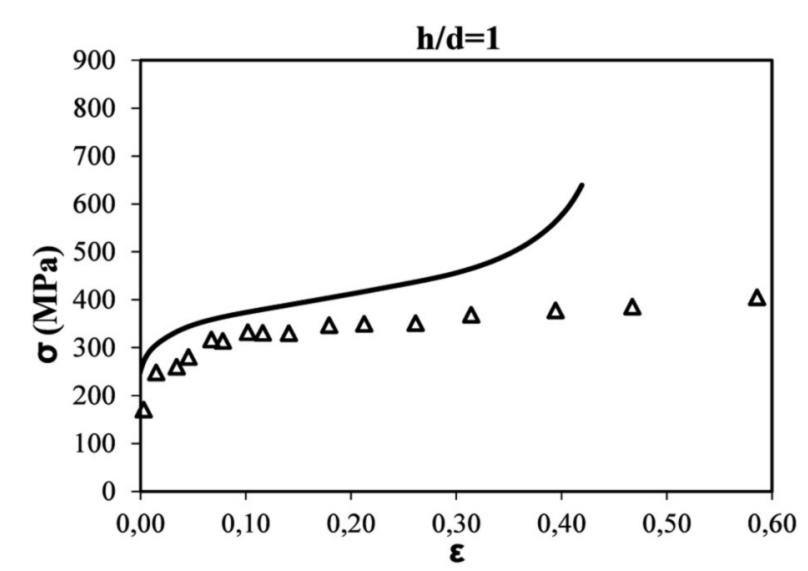

Continuo

$\Delta$ Incremental

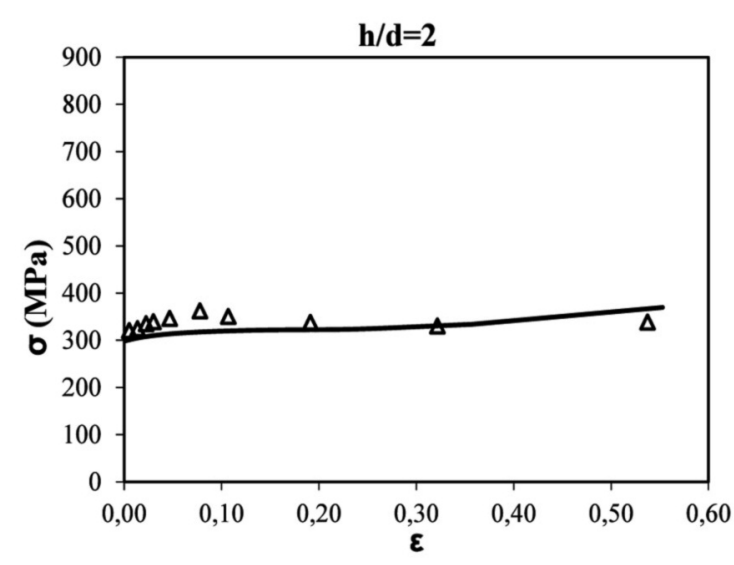

- Continuo

$\Delta$ Incremental

Figura 5. Curvas tensión-deformación obtenidas en los ensayos para diferentes factores de forma de la aleación AA 6082-T6.

Figure 5. Stress-strain curves obtained in the tests for different shape factors of AA 6082-T6 aluminum alloy. 
lizar en mayor detalle este fenómeno para un proceso de forja en frío simulado mediante el MEF bajo diferentes condiciones de rozamiento de tipo Coulomb $b^{[4]}$.

\subsection{Modelo de elementos finitos}

Se ha empleado el programa de elementos finitos de metodología explícita ABAQUS/Explicit ${ }^{[20]}$. En la modelización del proceso se han considerado las mismas condiciones de trabajo que en los ensayos experimentales realizados. Para ello se ha impuesto un desplazamiento axial de compresión sobre las piezas igual al que se ha alcanzado en cada uno de los ensayos realizados en función de la geometría de las mismas. Se han impuesto unas condiciones de rozamiento de tipo Coulomb, las cuales se han ido variando en las distintas simulaciones realizadas, desde $\mu=0,1$ hasta 0,5 en intervalos de 0,1 . Se ha supuesto que existe el mismo rozamiento en todas las superficies pieza-plato de compresión en contacto.

El modelo numérico ha sido desarrollado considerando la pieza como un cuerpo deformable y los platos de compresión como cuerpos rígidos, asumiendo condiciones axisimétricas. El cuerpo deformable, en este caso la pieza, ha sido caracterizada con una malla homogénea compuesta por elemen- tos continuos, de primer orden con integración reducida siendo los elementos del tipo CAX4R. En ABAQUS/Explicit, las condiciones de contorno y las cargas no se pueden admitir como instantáneas, se deben aplicar de manera gradual a través de una curva de amplitud, la cual se ha definido en función del tiempo.

El material introducido en el modelo numérico ha sido caracterizado mediante el ensayo de compresión uniaxial con aplicación de la carga de manera incremental. Esta metodología permite obtener una curva tensión-deformación lo más independiente posible de las condiciones de rozamiento del ensayo.

Como variables de salida se han registrado la fuerza necesaria para llevar a cabo el proceso y el desplazamiento de la matriz superior, siendo estas variables conocidas en los ensayos experimentales realizados. Así mismo, aprovechando la potencialidad de este tipo de herramientas, se han evaluado también las presiones de contacto en la intercara pieza-plato de compresión y las deformaciones plásticas equivalentes en las piezas al final del proceso de compresión.

Antes de pasar a analizar aspectos como la influencia del factor de forma y del rozamiento mediante el modelo numérico implementado, se procede a validar el mismo por comparación de los resultados obtenidos por el MEF con los extraídos de los experimentos rea-

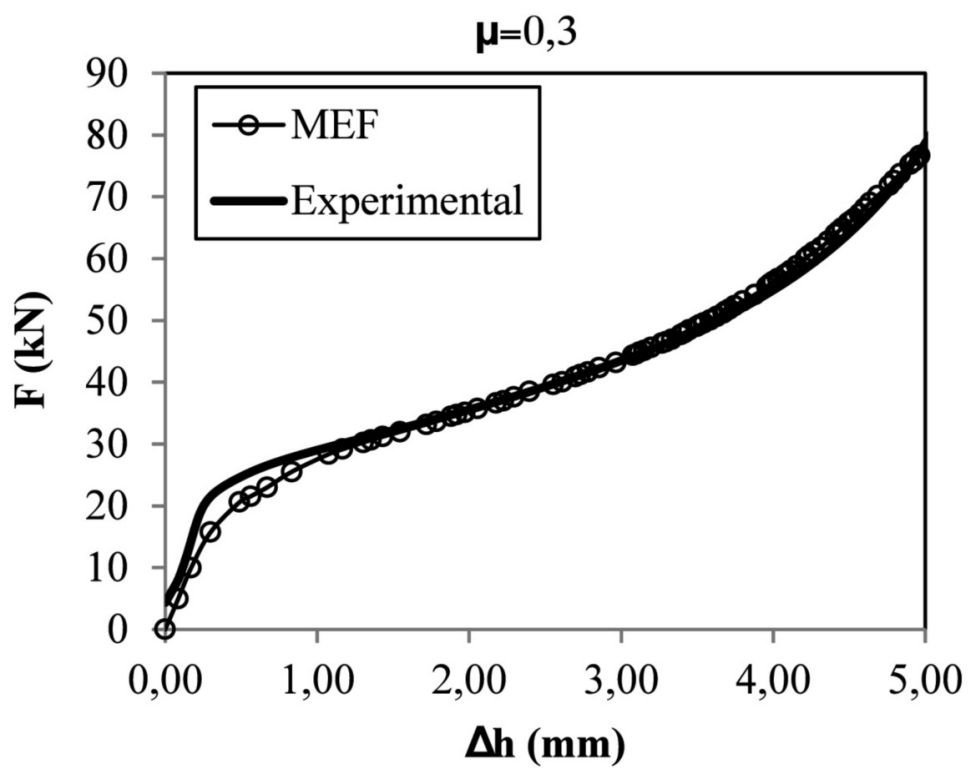

Figura 6. Validación de los resultados del modelo numérico con técnicas experimentales para un factor de forma $h / d=1$ de la aleación AA 6082-T6 con un rozamiento $\mu=0,3$.

Figure 6. Validation of the numerical results with experimental techniques for shape factor $h / d=1$ of AA 6082-T6 aluminum alloy with a friction coefficient $\mu=0.3$. 
lizados, obteniéndose valores prácticamente idénticos. En la figura 6 se muestran las gráficas obtenidas para un factor de forma $h / d=1$ considerando un coeficiente de rozamiento de Coulomb de 0,3 en la simulación mediante el MEF.

\section{ANÁLISIS Y DISCUSIÓN DE LOS RESULTADOS}

\subsection{Influencia del factor de forma}

Como se muestra en las gráficas de la figura 5 , la curva tensión-deformación incremental está por debajo de la curva tensión-deformación obtenida en el proceso con aplicación de la carga de compresión de manera continua, mientras que en el estudio de la pieza con un factor de forma $h / d=2$, ambas curvas son prácticamente idénticas.

En la figura 7 se han representado los resultados obtenidos de presiones frente a las deformaciones alcanzadas considerando diferentes factores de forma.

En dicha figura se muestran los resultados extraídos considerando un coeficiente de rozamiento de 0,3 para el caso de los factores de forma $(h / d): 0,5$ y 1 . Como se observa en las curvas, se alcanza mayores presiones en la pieza con un menor factor de forma mientras que las deformaciones máximas alcanzadas, en este caso, son menores. Por tanto, el factor de forma de la pieza influye en el estado tensional y deformacional alcanzado en la pieza después de someterla al proceso de compresión. Los resultados parecen indicar que en un proceso de compresión tipo forja abierta, para idéntica área de contacto (es decir, mismo diámetro inicial de la pieza) se registran mayores presiones totales cuando se conforman piezas con una relación de aspecto más achatada (es decir, para alturas menores).

En las figuras 8 y 9 se muestran los diagramas de deformaciones y tensiones obtenidos de las simulaciones realizadas mediante el MEF. En las figuras se ha representado el mismo modelo considerando dos factores de forma, 0,5 y 1 , con el mismo rozamiento, $\mu=0,3$, y en el mismo instante del proceso de compresión: se han extraído los resultados cuando la matriz superior se ha desplazado $2 \mathrm{~mm}$. Comparando los diagramas de deformaciones representados en la figura 6 se observa que se alcanzan mayores deformaciones cuanto menor es el factor de forma. Los valores máximos de deformación se producen en el centro de la pieza y extremos del área en contacto de la pieza con las matrices de compresión.

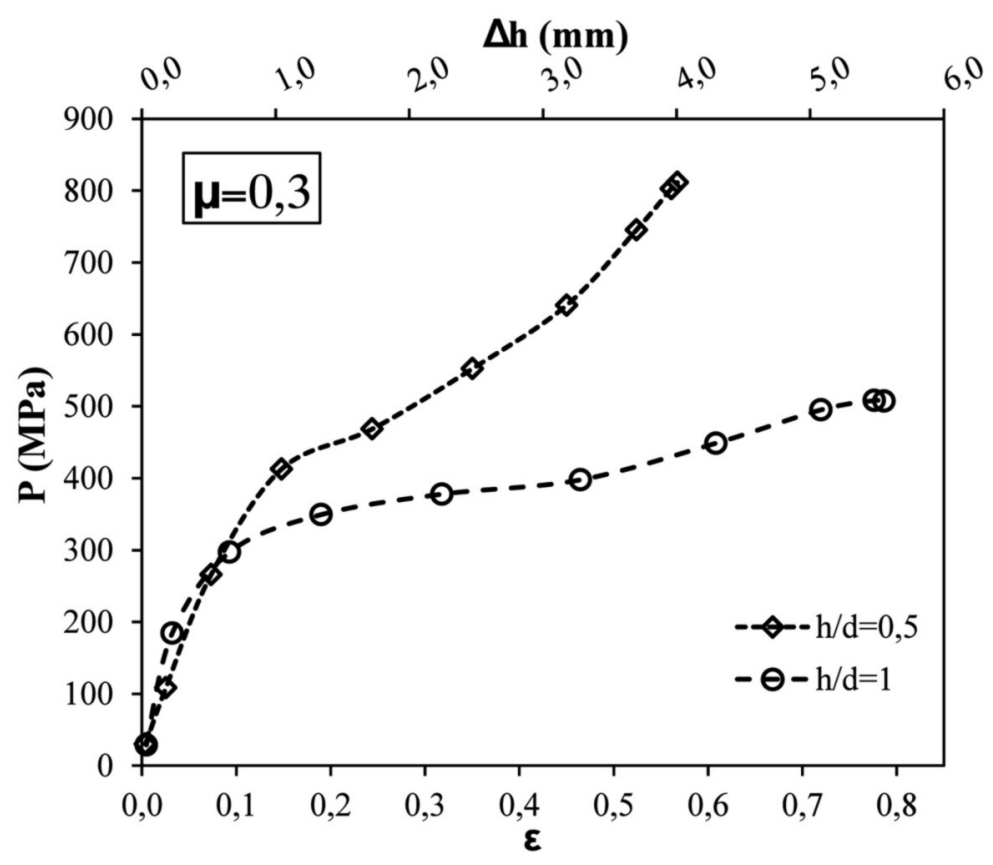

Figura 7. Influencia del factor de forma en las presiones aplicadas a compresión (aleación AA 6082-T6).

Figure 7. Influence of shape factor on compression pressures applied (AA 6082-T6 aluminum alloy). 


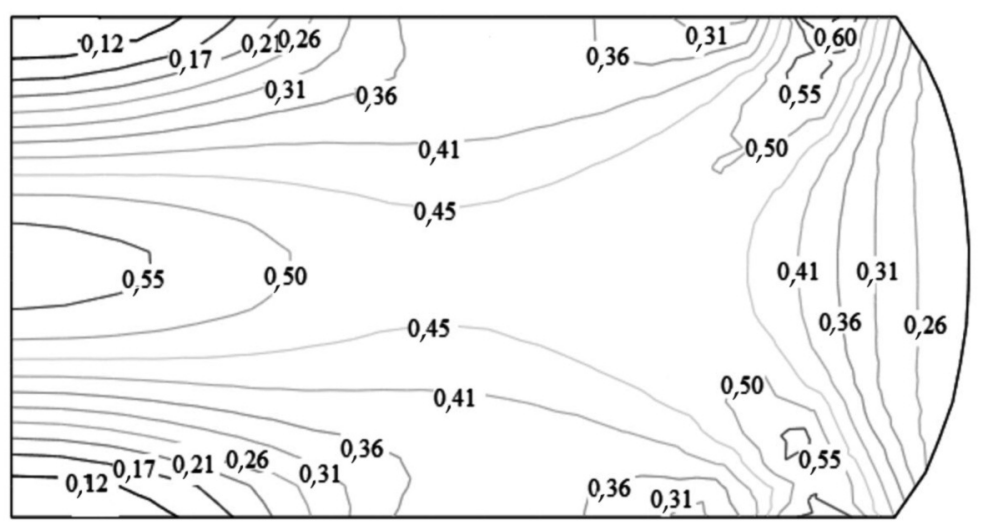

$h / d=0,5$

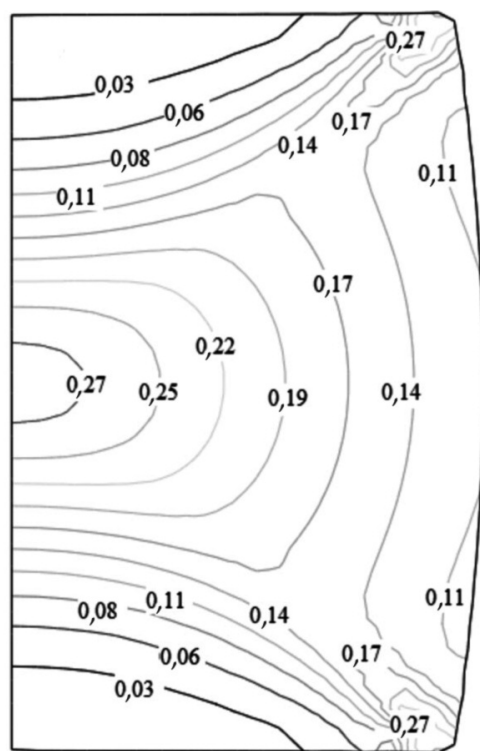

$h / d=1$

Figura 8. Diagramas de deformaciones equivalentes en piezas con diferente factor de forma considerando un rozamiento $\mu=0,3$ y con un $\Delta h=2 \mathrm{~mm}$.

Figure 8. Equivalent strain diagrams for workpieces with different shape factors, a friction coefficient $\mu=0.3$ and $\Delta h=2 \mathrm{~mm}$.

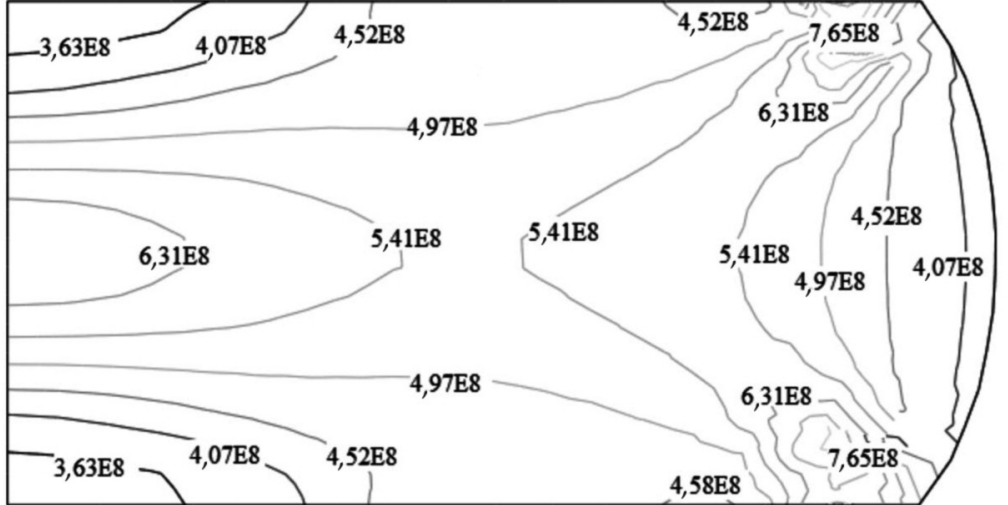

$h / d=0,5$

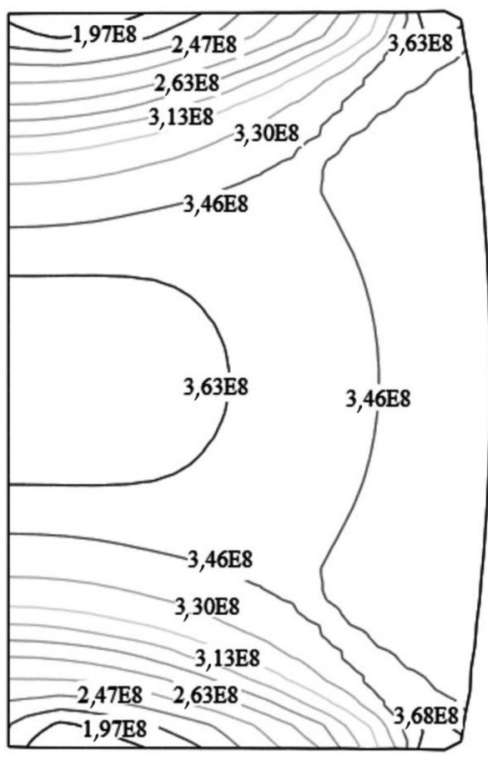

$h / d=1$

Figura 9. Diagramas de tensiones equivalentes en piezas con diferente factor de forma considerando un rozamiento $\mu=0,3$ y con un $\Delta \mathrm{h}=2 \mathrm{~mm}$.

Figure 9. Equivalent stress diagrams for workpieces with different shape factors, a friction coefficient $\mu=0.3$ and $\Delta h=2 \mathrm{~mm}$.

En la figura 9 se muestran las tensiones alcanzadas para el mismo modelo, observándose que se obtienen mayores tensiones en la pieza con un menor factor de forma. Al igual que en el caso de las deformaciones, los valores máximos de tensión se producen en el centro de la pieza y en los extremos del área de contacto. 
Hay que tener en cuenta que los resultados de deformaciones y tensiones son extraídos del mismo instante del proceso, $\Delta \mathrm{h}=2 \mathrm{~mm}$, alcanzándose un estado tensional y deformacional más elevado en la pieza con un menor factor de forma, mientras que en la pieza con un factor de forma mayor el estado tensional y deformacional alcanzado es menor, permitiendo someterse a una mayor deformación.

\subsection{Influencia del rozamiento}

Los resultados obtenidos para el material AA 6082-T6 en función de los diferentes coeficientes de rozamiento de Coulomb se han representado en la figura 10. Las gráficas de la figura representan las presiones totales aplicadas por las matrices frente a las deformaciones producidas durante el proceso de compresión considerando diferentes rozamientos. En las mismas gráficas se ha incluido una segunda escala en el eje de abscisas donde se reflejan también los incrementos de altura (decrementos de altura al ser un proceso de compresión).

Como se puede observar en las gráficas de esta figura, la influencia del rozamiento difiere en función del factor de forma de la pieza. En la pieza con factor de forma $h / d=0,5$ se observa que a medida que aumenta el rozamiento las tensiones son más elevadas. Esta tendencia es mayor cuanto mayor es la deformación, es decir, cuando se producen pequeñas deformaciones las curvas son prácticamente iguales; al aumentar estas deformaciones la diferencia entre las curvas crece. Sin embargo en la pieza con un factor de forma $h / d=1$, prácticamente el rozamiento no influye en las curvas, sólo a partir de una deformación superior a $\varepsilon=0,6$ se empiezan a diferenciar.

Este fenómeno guarda una estrecha relación con lo apuntado por Kopp y Wiegels ${ }^{[21]}$ en relación a la obtención de curvas de fluencia mediante ensayos de compresión, donde se indica que se debe a la relación geométrica entre la altura y el diámetro de la pieza a lo largo del proceso. Cuando el factor de forma de la pieza es menor que $0,5(h / d<0,5)$, hay una mayor influencia del rozamiento. En el caso en el que el factor de forma de la pieza es mayor o igual a $0,5(h / d \geq 0,5)$, no es tan importante la energía invertida en vencer el rozamiento respecto a otras contribuciones de energía del proceso y por tanto las curvas tensión-deformación no dependen del mismo. A medida que se aplica carga axial de compresión en la pieza, la altura y el diámetro de la misma van cambiando, por tanto la relación $h / d$ a lo largo del proceso se irá modificando de manera que va disminuyendo, entrando en el rango $h / d<0,5$.

Partiendo de este hecho, se puede realizar una comprobación del citado fenómeno a través del análisis mediante elementos finitos efectuado. Si se obtienen los factores de forma $h / d$ que se van produciendo en el transcurso de las simulaciones que se han llevado a cabo considerando diferentes rozamientos, se puede comprobar en qué instante del proceso se pasa a tener un factor de forma menor que $0,5(h / d<0,5)$ y por tanto, en qué instante el rozamiento empieza a tener una mayor importancia
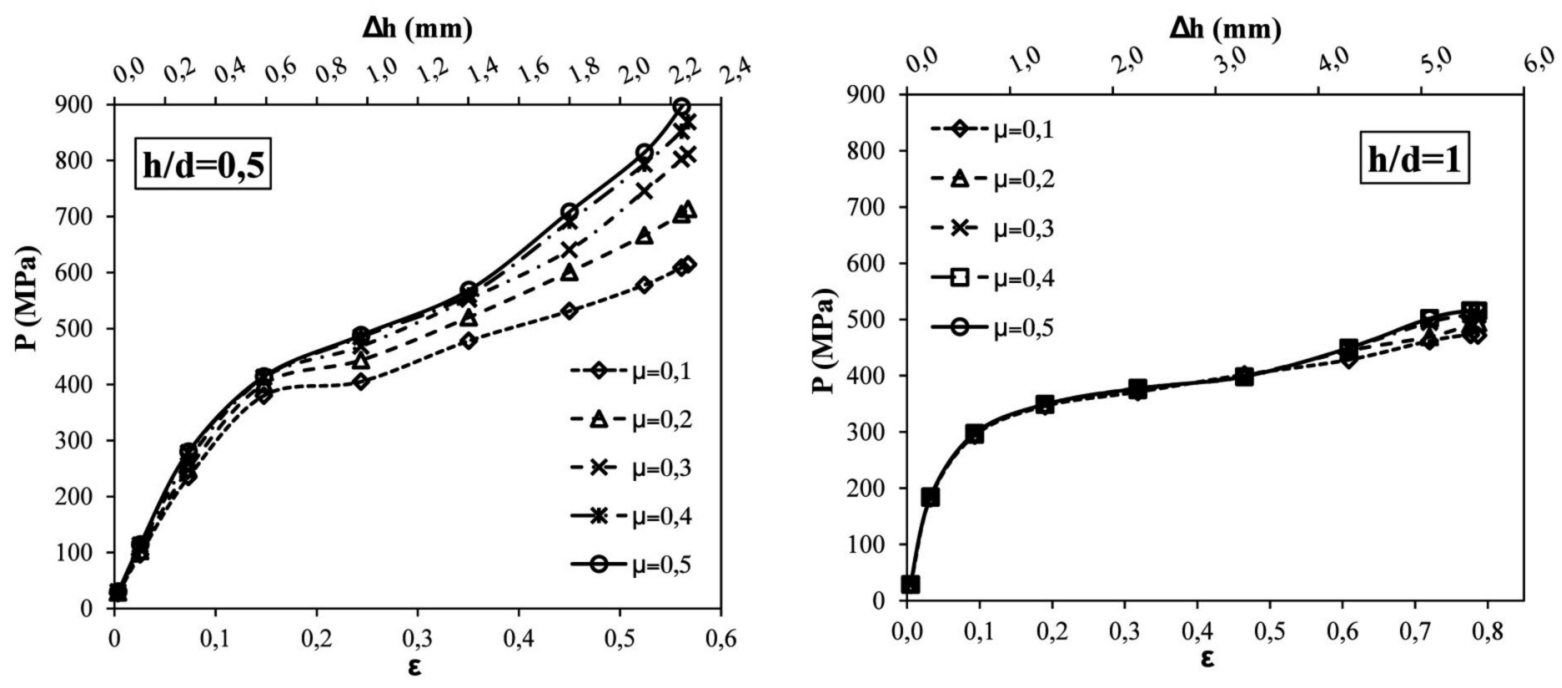

Figura 10. Influencia del rozamiento en curvas tensión-deformación a compresión para el material AA 6082-T6.

Figure 10. Influence of friction on compression pressures applied for two shape factors (AA 6082-T6 aluminum alloy). 
en el resultado de las fuerzas, lo que es de interés en el estudio de procesos de forja.

En la tabla I se muestran los factores de forma $h / d$ en cada incremento de altura, $\Delta \mathrm{h}$, de las simulaciones realizadas para el material AA 6082-T6. En la tabla se ha señalado el instante en el cual el factor de forma $h / d$ pasa a ser menor de 0,5 junto con el incremento de altura producido (decremento de altura en compresión). Tal como se ha mencionado anteriormente, en ese instante el rozamiento empieza a tener una mayor influencia en las fuerzas necesarias para llevar a cabo el proceso, comprobándose esta influencia en las curvas representadas en la figura 10 . Se observa cómo las curvas empiezan a diferenciarse unas de otras a partir de los incrementos de altura señalados en la tabla I.

Para una correcta determinación de las dimensiones finales o intermedias de la pieza obtenidas por experimentación, como por ejemplo el diámetro del plano medio en cada incremento de altura, es de especial utilidad el empleo de técnicas de escaneado tridimensional que permiten una mayor precisión de los resultados de la medida.

Siguiendo el planteamiento observado en cuanto a presiones aplicadas, sería de esperar un comportamiento similar también en las presiones de contacto. La falta de estudios en la literatura científica que aborde el análisis de este fenómeno en las presiones de contacto hace que resulte un campo particularmente interesante. Se han obtenido los perfiles de presión alcanzados en las superficies de contacto en función de los diferentes rozamientos considerados para cada una de las piezas ensayadas mediante el método de los elementos finitos.

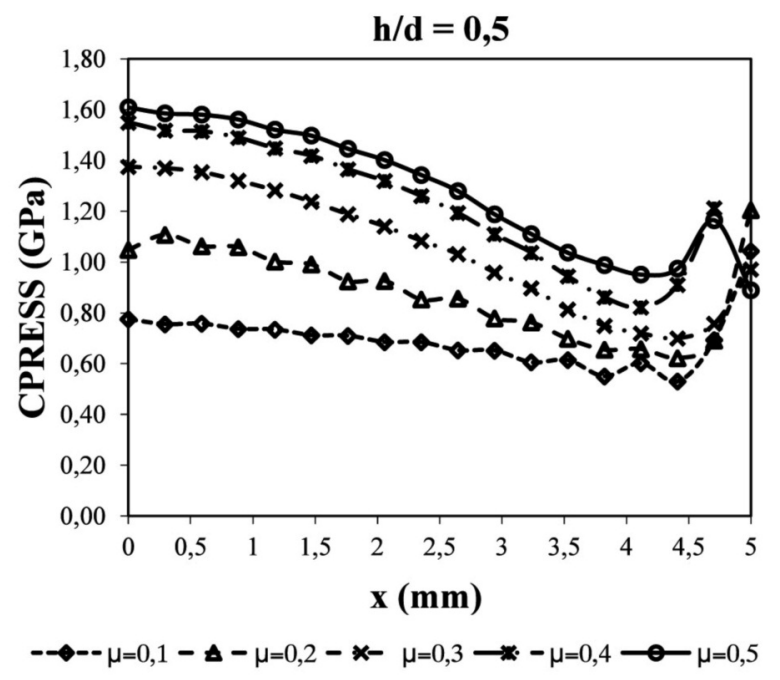

Tabla I. Relaciones $\mathrm{h} / \mathrm{d}$ de las piezas de AA 6082-T6 con su correspondiente incremento en altura obtenidas mediante simulación y determinación del punto de cambio de comportamiento

Table I. Ratios $h / d$ of the workpieces of AA 6082-T6 aluminum alloy obtained by FEM simulation, indicating their increase in height and definition of the point where a behavior change is observed

\begin{tabular}{cccc}
\hline \multicolumn{2}{c}{$\mathbf{h}_{\mathbf{0}} / \mathbf{d}_{\mathbf{0}} \mathbf{=} \mathbf{0 , 5}$} & \multicolumn{2}{c}{$\mathbf{h}_{\mathbf{0}} / \mathbf{d}_{\mathbf{0}}=\mathbf{1}$} \\
\hline $\mathbf{h} / \mathbf{d}$ & $\Delta \mathbf{h} \mathbf{( m m )}$ & $\mathbf{h} / \mathbf{d}$ & $\Delta \mathbf{h}(\mathbf{m m})$ \\
\hline 0,51 & 0 & 1,02 & 0 \\
0,51 & 0,02 & 1,01 & 0,05 \\
$\mathbf{0 , 4 9}$ & $\mathbf{0 , 1 3}$ & 0,97 & 0,32 \\
0,46 & 0,36 & 0,88 & 0,90 \\
0,41 & 0,70 & 0,77 & 1,75 \\
0,36 & 1,10 & 0,63 & 2,75 \\
0,30 & 1,50 & 0,51 & 3,75 \\
0,26 & 1,84 & $\mathbf{0 , 4 1}$ & $\mathbf{4 , 0 0}$ \\
0,23 & 2,07 & 0,35 & 5,18 \\
0,22 & 2,18 & 0,32 & 5,45 \\
0,22 & 2,20 & 0,31 & 5,50 \\
\hline
\end{tabular}

En la figura 11 se muestran los perfiles de presiones de contacto alcanzadas al final del proceso de compresión para las piezas con un factor de forma $(h / d)$ : 0,5 y 1 . Como se muestra en las gráficas obtenidas,

Figura 11. Influencia del rozamiento en la obtención de las presiones de contacto pieza-plato de compresión para la aleación AA 6082-T6.

Figure11. Influence of friction on contact pressure distributions for AA 6082-T6 aluminum alloy. 
los perfiles de presiones de contacto están influenciados por el factor de forma de la pieza. Para la pieza con un factor de forma $h / d=0,5$ se aprecia una mayor diferencia en los distintos perfiles de presión alcanzados para distintos rozamientos. A medida que aumenta el rozamiento se incrementan los valores de presiones en la superficie de contacto, mientras que en la pieza con un factor de forma de $h / d=1$ los perfiles de presiones son bastantes similares variando en un rango muy pequeño. Por otro lado, los perfiles de presiones para este factor de forma son mucho más suaves en comparación con los obtenidos en un factor de forma $h / d=0,5$ donde se muestran mucho más acentuados a medida que aumenta el rozamiento. $\mathrm{Al}$ comparar los perfiles de presión para un mismo rozamiento considerando diferente factor de forma se alcanzan valores de presiones de contacto mayores en las piezas con un menor factor de forma.

Por tanto, al igual que ocurre con las fuerzas/presiones aplicadas, los valores de los perfiles de presión alcanzados en las superficies de contacto están influenciados por el rozamiento existente, pudiéndose minimizar su efecto mediante la elección de un factor de forma de la pieza adecuado. Ello tendría una especial incidencia en la disminución del desgaste experimentado por las herramientas empleadas en procesos de forja abierta, y previsiblemente en procesos de estampación donde el diseño y mantenimiento de la integridad de las estampas desempeña un papel fundamental ${ }^{[22]}$, permitiendo un aumento de su vida en servicio. Por otro lado, sería de interés evaluar el estado superficial de las piezas sometidas a diferentes condiciones de presión en la superficie como consecuencia de este fenómeno, aun estando sometidas a idénticas condiciones de rozamiento.

\section{CONCLUSIONES}

- En el estudio de cualquier proceso es muy importante caracterizar el material y conocer su comportamiento bajo condiciones de trabajo dadas, aspecto especialmente crítico cuando se busca incorporar mejoras en el mismo. La caracterización del comportamiento de materiales metálicos a tracción uniaxial está muy extendida, siendo más habitual el empleo de los ensayos de compresión uniaxial para el estudio del comportamiento de materiales frágiles o poco dúctiles, como por ejemplo los cerámicos. No obstante, en los PCDPC las piezas están sometidas a grandes cargas de compresión generándose estados tensionales a compresión elevados. Debido a ello caracterizar el comportamiento del material bajo similares condiciones de trabajo permite abordar el proceso desde un punto de vista más realista. Debido a la variedad de procesos de fabricación que pertenecen al grupo de los PCDPC, en el presente estudio se ha optado por empezar por el proceso más sencillo, esto es, aplicación de una carga de compresión uniaxial a una pieza cilíndrica mediante matrices planas y paralelas simétricamente opuestas.

- Para el estudio del proceso de compresión se ha empleado como herramienta de análisis el MEF. En este tipo de herramientas es de vital importancia introducir todas las entradas de la manera más realista posible. Ello es de especial significación cuando se trata de introducir las propiedades mecánicas del material en cuestión, siendo más adecuado el empleo de curvas de compresión cuando se trabaja en el análisis de PCDPC. En este trabajo, las características del material han sido obtenidas mediante ensayos de compresión y se han utilizado en el modelo MEF empleado, habiéndose validado los resultados con los obtenidos experimentalmente, observándose una gran coincidencia entre resultados.

- A lo largo del artículo se han analizado parámetros tecnológicos, como el rozamiento y la geometría de la pieza, para conocer mejor el comportamiento del material metálico ante un proceso de compresión y en un futuro poderlo extender a otros procesos pertenecientes al grupo de los PCDPC más complejos, por ejemplo, la estampación.

- Como se ha podido ver a lo largo de este estudio, los resultados de las principales variables de los procesos de compresión mediante matrices planas y paralelas como fuerzas, presiones de contacto y tensiones dependen del rozamiento existente entre las superficies en contacto. Por tanto, habrá que minimizar su efecto. Esto se consigue bien aplicando el BCFM motivado por una aplicación de la carga de manera incremental durante el proceso de compresión, o bien eligiendo una geometría inicial adecuada de la probeta definida por su factor de forma.

- En el proceso de compresión mediante matrices planas y paralelas se ha demostrado que se debe elegir una geometría de pieza inicial con un factor de forma lo más elevado posible. Por otro lado, se ha comprobado que siempre que exista a lo largo del proceso un factor de forma $\mathrm{h} / \mathrm{d} \geq 0,5$, la fuerza obtenida en función del desplazamiento de la herramienta no depende del rozamiento entre las superficies en contacto.

- Se ha demostrado que eligiendo una adecuada geometría inicial de la probeta se minimiza el efecto del rozamiento en otras variables como tensiones y presiones de contacto, permitiendo además que se alcancen mayores deformaciones. 
Estos resultados presentan un interés especial para alcanzar una disminución del desgaste de las matrices de forja, permitiendo un aumento de su vida en servicio de las mismas. Por otro lado, se indica como una de las líneas futuras a desarrollar, el interés de evaluar el estado superficial de las piezas bajo diferentes condiciones de presión en la superficie aun estando sometidas a idénticas condiciones de rozamiento como consecuencia del fenómeno identificado en el artículo.

\section{Agradecimientos}

Este trabajo ha sido financiado a través del Proyecto de Investigación DPI2009-07300 concedido por el Ministerio de Ciencia e Innovación junto con las ayudas anuales de la E.T.S. de Ingenieros Industriales de la Universidad Nacional de Educación a Distancia (UNED).

\section{REFERENCIAS}

[1] DIN 8582: 2003, Manufacturing processes forming-Classification, subdivision, terms and definitions, alphabetical index, Berlín, Germany, 2003.

[2] DIN 8585-3: 2003, Manufacturing processes forming under compressive conditions - Part 3: Free forming; Classification, subdivision, terms and definitions, Berlín, Germany, 2003

[3] T. Altan, G. Ngaile y G. Shen, ASM International, Columbus, OH, EE.UU., 2005.

[4] M.M. Marín, Tesis Doctoral, Universidad Nacional de Educación a Distancia (UNED), Madrid, España, 2011.

[5] S. Diot, D. Guines, A. Gavrus y E. Ragneau, J. Eng. Mater. Technol. 131 (2009) 1-10.
[6] R. Ebrahimi y A. Najafizadeh, J. Mater. Process. Tech.152 (2004) 136-143.

[7] D. Szeliga, J. Gawad y M. Pietrzyk, Comput. Method. Appl. 195 (2006) 6.778-6.798.

[8] H. Sofuoglu y H. Gedikli, Tribol. Int. 35 (2002) 27-34.

[9] X. Tan, Tribol. Int. 35 (2002) 385-393.

[10] J.P. Wang, J. Mater. Process. Tech. 123 (2002) 323-328.

[11] E.M. Rubio, R. Domingo, C. González y A. Sanz, Rev. Metal. 40 (2004) 90-100.

[12] F. Fereshteh-Saniee, I. Pillinger y P. Hartley, J. Mater. Process. Tech. 153-154 (2004) 151156.

[13] A.M. Camacho, M.M. Marín, L. Sevilla y C. Bernal, Proc. ICADME, Taiyuan, China, 2012.

[14] M.M. Marín, A.M. Camacho, E.M. Rubio y M.A. Sebastián, Proc. ESM, C. Bertelle y A. AyeshGreen (Eds), EUROSIS, Ostende, Bélgica, 2008, pp. 51-55.

[15] A.M. Camacho, C. Vallellano, F.J. GarcíaLomas y M.A. Sebastián, Steel Res. Int. 81 (2010) 958-961.

[16] F. Fereshteh-Saniee y F. Fatehi-Sichani, J. Mater. Process. Tech. 177 (2006) 478-482.

[17] P. F. Bariani, T. Dal Negro y S. Bruschi, CIRP Annals - Manuf. Techn. 53 (2004) 573-595.

[18] A. Duckham y R. D. Knutsen, Mat. Sci. Eng. A-Struct. 256 (1998) 220-226.

[19] T. Altan, S.I. Oh y H.L. Gegel, ASM International, Columbus, OH, EE.UU., 1983.

[20] D. Hibbitt, B. Karlsson y P. Sorensen, ABAQUS v6.10, User's Manuals, Simulia, Providence, RI, EE.UU., 2010.

[21] R. Kopp y H. Wiegels, Einführung in die umformtechnick, Institut für Bildsame Formgebung, Ed. Mainz, G., Aachen, Germany, 1999.

[22] J.M. Arenas, M.A. Sebastián, P. Núñez y C. González, Rev. Metal. 36 (2000) 366-374. 